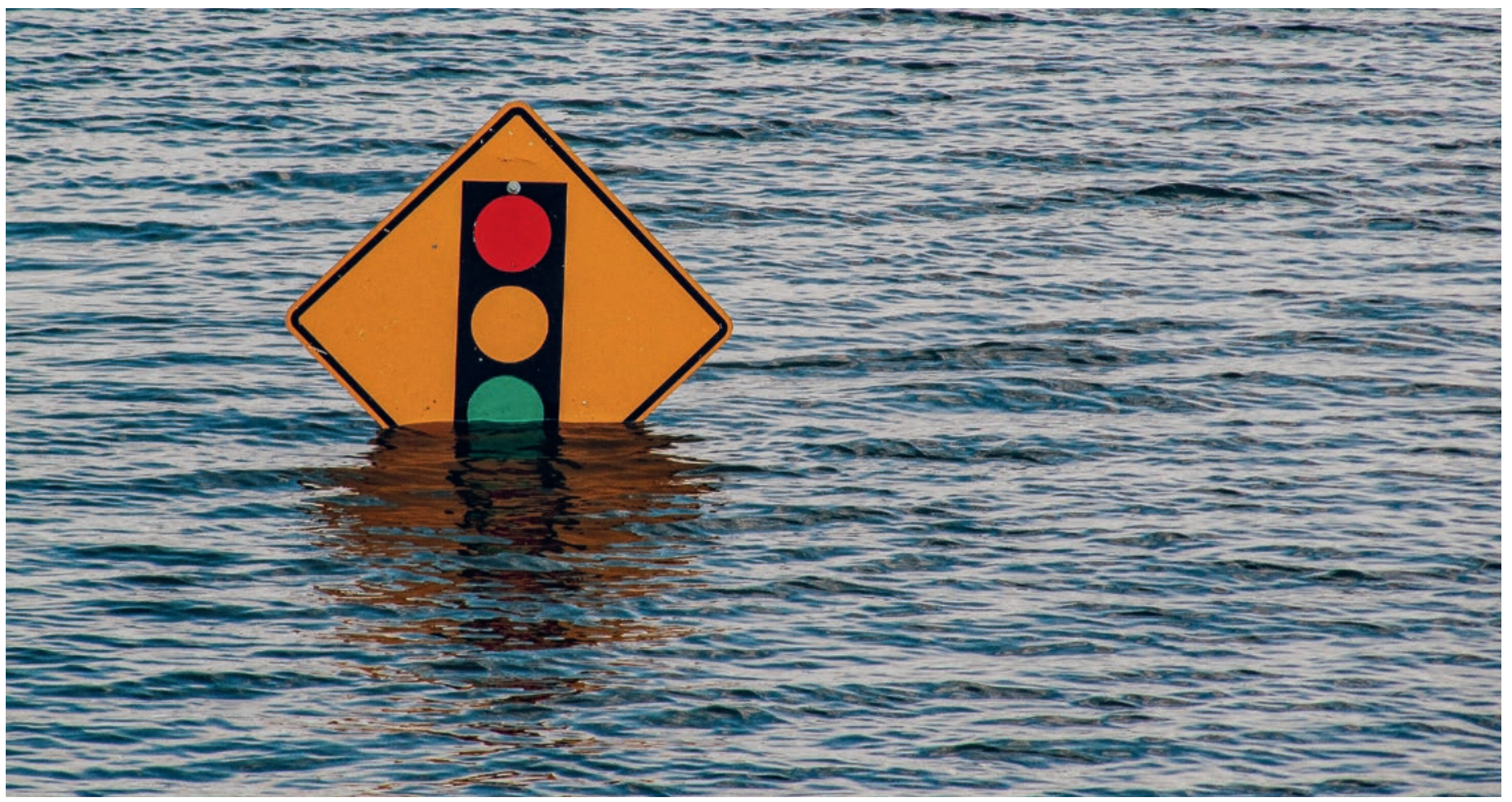

\title{
Strategien gegen unzulässige Methoden von Krankenversicherern
}

\section{Andrea Schütz}

Dr., LL.M., Associated Partner, Prager Dreifuss AG, Zürich

Die Gesundheitskosten in der Schweiz sind hoch und steigen weiter an. Der Kostendruck darf jedoch nicht dazu führen, dass Krankenversicherer ihre Kompetenzen überschreiten und sich unter dem Deckmantel der Wirtschaftlichkeitskontrolle unzulässiger Methoden bedienen. Nachfolgend werden unrechtmässige Vorgehensweisen von Krankenversicherern aufgezeigt und es wird dargelegt, wie sich Ärzte dagegen zur Wehr setzen können.

Die zulasten der obligatorischen Krankenpflegeversicherung (OKP) abgerechneten Leistungen müssen wirksam, zweckmässig und wirtschaftlich sein [1]. Somit haben sich Ärzte in ihren Leistungen auf das Mass zu beschränken, das im Interesse der Versicherten liegt und für deren Behandlung erforderlich ist. Für Leistungen, die über dieses Mass hinausgehen, kann die Vergütung verweigert bzw. zurückgefordert werden. Das Gesetz schreibt vor, dass Leistungserbringer und Versicherer "vertraglich» eine Methode zur Wirtschaftlichkeitskontrolle festlegen [2].

\section{Umgehung der vertraglich vereinbarten Screening-Methode}

Santésuisse und curafutura, denen die Mehrheit der Krankenversicherer angeschlossen ist, sowie die FMH haben sich 2013 beziehungsweise 2014 auf die ANOVAMethode verständigt. Sie vereinbarten aber bereits damals, dass die ANOVA-Methode weiterentwickelt werden soll. Diese Weiterentwicklung stellt die im Jahr 2018 vertraglich vereinbarte Screening-Methode dar. Die Screening-Methode erfolgt auf Basis des Daten- 
und Tarifpools der SASIS AG und kommt in allen Verfahren vor sämtlichen Instanzen ab dem Statistikjahr 2017 zur Anwendung [3].

Es gibt jedoch Krankenversicherer, die bei Wirtschaftlichkeitskontrollen auf eigene Datenpools und Methoden abstellen, obwohl sie der santésuisse bzw. der curafutura angehören. Sie behaupten, dass sie trotz des abgeschlossenen Vertrags bezüglich der ScreeningMethode «frei» seien, eigene systematische Wirtschaftlichkeitskontrollen durchzuführen.

Diese Behauptung ist unzutreffend, denn Art. 56 Abs. 6 KVG schreibt ausdrücklich vor, dass Leistungserbringer und Versicherer "vertraglich" eine Methode zur Wirtschaftlichkeitskontrolle festlegen. Diese Bestimmung bezweckt, die Wirtschaftlichkeitskontrolle für Ärzte transparent und nachvollziehbar zu machen und damit eine beidseitige Akzeptanz für die anzuwendende Methode zu schaffen [4].

Ärzte können sich deshalb gegenüber solchen Krankenversicherern zur Wehr setzen und darauf bestehen, dass diese ab dem Statistikjahr 2017 in allen Verfahren die Screening-Methode aus dem Jahr 2018 auf Basis des Datenpools der SASIS AG anwenden.

\section{«Nötigung» zum Abschluss von nicht genehmigten Tarifverträgen}

Das Gesetz schreibt vor, dass Tarife und Preise in Verträgen zwischen Versicherern und Leistungserbringern $\mathrm{zu}$ vereinbaren sind. Es gilt der Grundsatz der Tarifautonomie. Parteien eines Tarifvertrages sind einzelne oder mehrere Leistungserbringer oder deren Verbände einerseits sowie einzelne oder mehrere Versicherer oder deren Verbände anderseits. Ist ein Verband Vertragspartei, so ist der Tarifvertrag für die Mitglieder des Verbandes nur dann verbindlich, wenn sie ihm beigetreten sind. Das Gesetz will verhindern, dass einzelne Ärzte zu einem Vertragsschluss gezwungen werden [5].

Ärzte können darauf bestehen, dass Krankenversicherer ab dem Statistikjahr 2017 in allen Verfahren die Screening-Methode aus dem Jahr 2018 auf Basis des Datenpools der SASIS AG anwenden.

Sodann wird gesetzlich festgehalten, dass Tarifverträge der Genehmigung durch die zuständige Behörde bedürfen. Je nach Geltungsbereich des Vertrages ist dies die Kantonsregierung oder der Bundesrat. Die Genehmigungsbehörde prüft, ob der Tarifvertrag mit dem Gesetz und dem Gebot der Wirtschaftlichkeit und Billigkeit in Einklang steht. Dabei wird auch auf eine betriebswirtschaftliche Bemessung und eine sachge- rechte Struktur der Tarife geachtet [6]. Erst die Genehmigung des Tarifvertrages durch die zuständige Behörde hat rechtserzeugende Wirkung [7].

In der Praxis kommt es jedoch vor, dass Krankenversicherer Ärzte, welche korrekt über TARMED abrechnen, wegen vermeintlicher Unwirtschaftlichkeit über Monate hinweg mit hohen und nicht gerichtlich überprüften Rückforderungssummen konfrontieren und diesen anschliessend "nahelegen", einem nicht genehmigten Tarifvertrag mit Pauschalabrechnung zuzustimmen, da die Einzelfallkontrolle doch für beide Parteien sehr aufwendig sei.

Der Tiers garant kann für Ärzte bezüglich der Wirtschaftlichkeitskontrolle von Vorteil sein, da im Tiers garant der Versicherte und nicht der Krankenversicherer Schuldner der Leistung ist.

Ärzten in dieser Situation ist anzuraten, sich gegenüber den Krankenversicherern auf die Tarifautonomie zu berufen und diese darauf hinzuweisen, dass sie nicht zum Abschluss eines Tarifvertrages gezwungen werden können. Betroffene Ärzte sollten den Krankenversicherern auch mitteilen, dass Tarifverträge, welche nicht durch die zuständige Genehmigungsbehörde überprüft und genehmigt wurden, von vornherein nicht rechtverbindlich sind.

\section{«Nötigung» zum Wechsel auf das System des Tiers payant}

Die OKP kennt im ambulanten Bereich zwei Arten von ärztlichen Rechnungsstellungen, den Tiers garant und den Tiers payant. Obwohl der Tiers garant im ambulanten Bereich als Regelfall gilt, ist der Tiers payant gesetzlich nicht ausgeschlossen und kann vertraglich zwischen den Leistungserbringern und Versicherern vereinbart werden [8].

Grundsätzlich haben beide Abrechnungsmethoden ihre Vor- und Nachteile. Die FMH hat sich jedoch für den Tiers garant als Standardmodell ausgesprochen. Sie begründete dies insbesondere damit, dass den $\mathrm{Pa}$ tienten beim Tiers garant eine aktivere Rolle zukommt, da sie selber entscheiden können, welche Rechnungen sie ihren Versicherern zur Vergütung einreichen. Auch dem Daten- und Persönlichkeitsschutz der Patienten wird nur im Tiers garant vollständig Rechnung getragen, denn sie können nur beim Tiers garant entscheiden, welche Informationen die Versicherer über ihre Veranlagungen, Krankheiten oder Eingriffe erhalten [9].

Der Tiers garant kann für Ärzte aber auch bezüglich der Wirtschaftlichkeitskontrolle von Vorteil sein, da 
im Tiers garant der Versicherte und nicht der Krankenversicherer Schuldner der Leistung ist. Während im Tiers payant die Krankenversicherer Rechnungen für Leistungen, die in ihren Augen nicht wirtschaftlich sind, nach Bestreitung einfach unbezahlt lassen können [10], fehlt dieses Druckmittel im System Tiers garant. Zwar besteht auch beim Tiers garant das Risiko, dass Patienten vereinzelt Rechnungen nicht begleichen. Dies stellt jedoch das kleinere Übel dar, als wenn Krankenversicherer eine Vielzahl von Rechnungen wegen vermeintlicher Unwirtschaftlichkeit unbezahlt lassen.

Dass sich Krankenversicherer dieses Druckmittels durchaus bewusst sind, ergibt sich aus einer vom BAG in Auftrag gegebenen Untersuchung über die Rechnungs- und Wirtschaftlichkeitskontrolle bei Krankenversicherern. Anlässlich dieser Untersuchung haben Krankenversicherer moniert, dass es im Tiers garant an "effektiven Sanktionsmöglichkeiten" gegenüber den Leistungserbringern fehlen würde [11]. Krankenversicherer dürften damit die fehlende Möglichkeit zur Verweigerung der Bezahlung von Rechnungen vor Augen gehabt haben, denn sie können auch im System Tiers garant Rückerstattungsforderungen beim Schiedsgericht einreichen [12]. Die gerichtliche Überprüfung der Frage der Wirtschaftlichkeit in einem unabhängigen und fairen Verfahren ist jedoch für sie viel aufwendiger als die Nichtbegleichung von vermeintlich zu hohen Rechnungen.

Wenn Krankenversicherer Ärzte bezüglich der Vorteile des Tiers payant «überzeugen» wollen - etwa mit dem Argument, dass dies im Sinne einer «besseren Kundenorientierung" sei-, so sollten sich diese bewusst sein, dass sie mit einem Wechsel auf Tiers payant den Krankenversicherern ein erhebliches finanzielles Druck-

\section{Krankenversicherer können Ärzte im System} Tiers payant relativ einfach zu kostengünstigen Abrechnungen «erziehen".

mittel an die Hand geben. Ärzte, die dies verhindern wollen, können den Krankenversicherern mitteilen, dass sie zu einem Wechsel auf Tiers payant nicht gezwungen werden können, da nicht der Tiers payant, sondern der Tiers garant der gesetzlich vorgesehene Regelfall ist [13].

\section{Umgehung des Schiedsgerichts durch angebliche "Verrechnungen»}

Wie oben aufgezeigt, können Krankenversicherer Ärzte im System Tiers payant relativ einfach zu kostengünstigen Abrechnungen «erziehen», indem sie ver- meintlich zu hohe Rechnungen einfach unbezahlt lassen. Sofern sie diese jedoch bereits bezahlt haben, können sie die gänzliche oder teilweise Rückerstattung nur noch klageweise beim zuständigen Schiedsgericht geltend machen [14].

Es gibt jedoch Krankenversicherer, die Rechnungen für neue und offensichtlich wirtschaftliche Leistungen (zum Beispiel Leistungen, die einem genehmigten Tarifvertrag entsprechen) von Ärzten unbezahlt lassen, weil sie in der Vergangenheit Rechnungen für Leistungen derselben Ärzte beglichen haben, welche sie nachträglich als zu hoch erachten. So hat ein Krankenversicherer behauptet, er würde «im Sinne einer administrativ vereinfachten Lösung» die Rechnungen für offene und unbestrittene Leistungen eines Arztes erst dann wieder bezahlen, wenn der aufgelaufene Totalbetrag den von ihm geschätzten und nicht gerichtlich überprüften Rückforderungsbetrag erreicht hat.

\section{Krankenversicherer müssen auch im Interesse} eines funktionierenden Gesundheitswesens in ihre rechtsstaatlichen Schranken verwiesen werden.

Im KVG-Bereich besteht jedoch keine gesetzliche Grundlage für eine solch "vereinfachte Lösung», welche darin besteht, wahllos Rechnungen für unbestrittene Leistungen über Monate hinweg unbezahlt zu lassen, bis ein vermeintlicher Rückforderungsbetrag, der nie gerichtlich überprüft und bestätigt wurde, erreicht wird. Mit diesem Vorgehen wird das gesetzlich vorgeschriebene Verfahren, wonach Krankenversicherer für vermeintlich unwirtschaftliche Leistungen eine Rückforderungsklage beim Schiedsgericht einreichen müssen, ausgehebelt. Krankenversicherer können dadurch selber "Gericht spielen", indem sie faktisch selber über die Wirtschaftlichkeit bzw. Unwirtschaftlichkeit der ärztlichen Leistung sowie über die angebliche Rückforderungssumme bestimmen.

Es geht jedoch nicht an, dass Krankenversicherer eigenmächtig über die Wirtschaftlichkeit bzw. Unwirtschaftlichkeit von ärztlichen Leistungen befinden und dadurch das gesetzlich vorgeschriebene Verfahren vor Schiedsgericht ausgehebelt wird. Dies gilt umso mehr, als die Vorgehensweise bei Abrechnungen unter TARMED auch gegen tarifvertragliche Verpflichtungen verstösst, da Krankenversicherer Rechnungen innerhalb einer bestimmten Frist zu bestreiten haben. Wurde diese Frist nicht gewahrt, gelten die entsprechenden Rechnungen als unbestritten und müssen innerhalb der vorgesehenen Zahlungsfristen bezahlt werden [15].

Ärzte, welche von solchen unzulässigen «Verrechnungen» betroffen und dadurch in eine finanzielle Not- 
situation geraten sind, können von sich aus das Schiedsgericht anrufen und diese unzulässige Vorgehensweise mittels vorsorglicher Massnahmen unterbinden. Sie müssen vor Gericht ihre drohende Zahlungsunfähigkeit nachweisen und beantragen, dass die Krankenversicherer ihre Rechnungen für unbestrittene Leistungen zulasten der OKP innerhalb der tarifvertraglichen Zahlungsfristen zu bezahlen haben. Selbst wenn in den einschlägigen kantonalen Gesetzen keine Regelung bezüglich des Erlasses von vorsorglichen Massnahmen im Verfahren vor Schiedsgericht zu finden sind, können solche Massnahmen beantragt und erlassen werden [16].

\section{Ärzte sollten sich konsequent zur Wehr setzen}

Die Bundesverfassung schreibt vor, dass Personen, die staatliche Aufgaben wahrnehmen, an die Grundrechte gebunden sind. Zugleich wird darin festgehalten, dass Grundlage und Schranke staatlichen Handels das Recht ist [17]. Krankenversicherer sind als Träger staatlicher Aufgaben im Bereich der OKP an das Gesetz gebunden und müssen bei der Ausführung ihres verfassungsmässigen Auftrags rechtsstaatliche Grundsätze beachten [18].

Die erwähnten Beispiele verdeutlichen jedoch, dass sich nicht alle Krankenversicherer dieser verfassungsmässigen Schranken vollumfänglich bewusst sind. Bedenklich ist insbesondere die erkennbare Tendenz einzelner Krankenversicherer, das Schiedsgericht zu umgehen und damit Ärzte, ohne dass die Frage der Wirtschaftlichkeit je gerichtlich geklärt wurde, in eine finanzielle Notlage zu versetzen.

Ärzten und Fachgesellschaften ist deshalb anzuraten, sich gegen unzulässige Verhaltensweisen von Krankenversicherern konsequent zur Wehr zu setzen. Ansonsten kann sich ein System etablieren, in dem Krankenversicherer eigenmächtig über die Wirtschaftlichkeit beziehungsweise Unwirtschaftlichkeit von ärztlichen Leistungen befinden können, obwohl diese Kompetenz allein den zuständigen Gerichten zukommt.

Zudem greift diese bedenkliche Entwicklung in die Therapiefreiheit der Ärzte ein, denn diese werden zur Vermeidung des Vorwurfs der Unwirtschaftlichkeit ihren Patienten nur noch die günstigsten, nicht aber die wirksamsten und zweckmässigsten Therapien anbieten. Diese Fehllenkung wird unweigerlich dazu führen, dass die Qualität der Gesundheitsversorgung sinkt [19].
Krankenversicherer müssen somit, nicht nur zum Wohl der Ärzteschaft und deren Patienten, sondern auch im Interesse eines funktionierenden Gesundheitswesens, in ihre rechtsstaatlichen Schranken verwiesen werden.

\section{Literatur}

1 Art. 32 Abs. 1 KVG

2 Art. 56 KVG.

3 Vertrag betreffend die Screening-Methode im Rahmen der Kontrolle der Wirtschaftlichkeit gemäss Art. 56 Abs. 6 KVG (Version vom 20.3.2018), abrufbar unter: www.fmh.ch/themen/ ambulante-tarife/wirtschaftlichkeitspruefung.cfm

4 BGE 144 V 79 ff., 82, E. 5.3.

5 Art. 43 und 46 KVG.

6 Art. 43 und Art. 46 KVG.

7 BVGer, C-536/2009, E. 6.5.3.

8 Art. 42 Abs. 1 und 2 KVG.

9 Gähler E, Irène Marty I, Schutz K. Die FMH für den Tiers garant. Schweiz Ärzteztg. 2014;95(5):147-9.

10 Art. 56 Abs. 2 KVG.

11 Untersuchung zur Rechnungs- und Wirtschaftlichkeitskontrolle bei Krankenversicherern, Bericht im Auftrag des BAG vom 23.1.2020, abrufbar unter: www.bag.admin.ch/bag/de/home/ versicherungen/krankenversicherung/kostendaempfung-kv.html 12 Art. 56 Abs. 2 lit. b und Art. 89 Abs. 3 KVG.

13 Art. 42 Abs. 1 KVG.

14 Art. 56 und Art. 89 KVG.

15 Art. 11 Abs. 10 Rahmenvertrag TARMED.

16 BVGer B-860/2011, E. 4.2.

17 Art. 35 Abs. 2 und Art. 5 Abs. 1 BV.

18 BGer 1C 372/2014, E. 6.

19 Romanens M, Kurth F, Warmuth W. Gefährliche Fehllenkung durch Wirtschaftlichkeitsverfahren. Schweiz Ärzteztg. 2018;99(10):324-7.

Bildnachweis

Unsplash/Kelly Sikkema

\section{Das Wichtigste in Kürze}

- Krankenversicherer, die der santésuisse bzw. der curafutura angehören, müssen bei der Wirtschaftlichkeitskontrolle die Screening-Methode auf Basis des Datenpools der SASIS anwenden.

- Der Tiers garant ist im ambulanten Bereich der gesetzlich vorgesehene Regelfall. Ärzte können nicht zum Tiers payant gezwungen werden.

- Krankenversicherer sind nicht befugt, eigenmächtig über die Wirtschaftlichkeit von ärztlichen Leistungen zu befinden.

\section{L'essentiel en bref}

- Les assureurs-maladie membres de santésuisse ou de curafutura doivent utiliser la méthode de screening basée sur le pool de données SASIS pour le contrôle de l'économicité

- Dans le secteur ambulatoire, le tiers garant est le cas standard prévu par la loi.

- Les assureurs-maladie ne sont pas autorisés à statuer de leur propre chef sur l'économicité des prestations médicales. 